\title{
Sheath Thickness Evaluation for Collisionless or Weakly Collisional Bounded Plasmas
}

\author{
Shiang-Bau Wang and Amy E. Wendt
}

\begin{abstract}
The widely used Child-Langmuir law for sheath thickness evaluation in semi-infinite collisionless plasmas makes the assumptions of quasi-neutrality $\left(n_{e}=n_{i}\right)$ and zero electric field intensity $E=0$ at the sheath edge, as well as applying the Bohm criterion for ions entering the sheath. However, through a whole region fluid model, Poisson's equation has been solved numerically for the steady-state solution through the sheath and presheath without these assumptions. With the sheath edge defined, as in the Child-Langmuir law, at the place where the ion velocity is equal to the Bohm velocity, the sheath thickness of a bounded collisionless or weakly collisional plasma has been found with this model in some cases to be much larger than that obtained with the Child-Langmuir Law. The sheath thickness discrepancy is significant under conditions found in low pressure high density plasma (HDP) tools for plasma processing. Results presented indicate that the sheath thickness is very sensitive to the electric field and space charge density at the sheath edge. The electric field and space charge density can be successfully estimated by an intermediate scale matching method [1]-[5], and are used to derive a modified expression for the potential in the sheath that can be solved numerically for sheath thickness. With these results, the matching problem, arising when sheath and plasma are modeled separately, can be overcome.
\end{abstract}

Index Terms - Modeling, plasma measurements, plasmas, plasma sheaths, space charge.

\section{INTRODUCTION}

$\mathbf{S}^{\mathrm{H}}$ HEATH thickness evaluation is an important issue for plasma diagnostics such as the widely-used Langmuir probe and for plasma etching in semiconductor manufacturing. To obtain the precise ion saturation current and therefore the ion density in plasmas from the I-V trace of Langmuir probe, analysis of the ion saturation portion of the I-V curve requires consideration of the edge effects which depend on sheath thickness [6]. This becomes essential in the situations where the plasma density cannot be evaluated by the electron saturation current. This includes, for example, electron cyclotron resonance (ECR) plasmas where noise is induced in the electron saturation region, electronegative plasmas and plasmas with small volume and/or small grounded wall area which are apt to be perturbed by a probe drawing high current in electron saturation. For plasma etching, especially for high

Manuscript received November 11, 1998; revised May 14, 1999. This work was supported by the Center for Plasma-Aided Manufacturing and NSF under Grant EEC8 721545.

The authors are with the Department of Electrical and Computer Engineering and Center for Plasma-Aided Manufacturing, University of Wisconsin Madison, Madison, WI 53706 USA (e-mail: wangs@ @ae.wisc.edu).

Publisher Item Identifier S 0093-3813(99)08110-2. aspect ratio cases, the etched feature profile is sensitive to the sheath thickness. The thicker the sheath, the more time the ions need to transit the sheath and hence the larger the chance of scattering collisions. Such collisions in the sheath region broaden the distribution of incident angles of ions bombarding the substrate and as a result are detrimental to anisotropic etching.

The semiconductor industry is currently developing and using high density plasma (HDP) tools for plasma etching. The typical discharge parameters are neutral pressure $\leq 30 \mathrm{mtorr}$, plasma density $10^{11}-10^{12} \mathrm{~cm}^{-3}$, and electron temperature $T_{e} \approx 3-4 \mathrm{eV}$. Under these conditions, the Debye length at the sheath edge is in the range $\lambda_{D s} \leq 5 \times 10^{-5} \mathrm{~m}$ and the mean free path for ions is around $\geq 1 \times 10^{-3} \mathrm{~m}$ for argon plasma (mass 40 amu., total ion-atom scattering cross section $10^{-14} \mathrm{~cm}^{2}$ for ion temperature $T_{i}=0.05 \mathrm{eV}$ [7]). Since the sheath thickness is at most several ten $\lambda_{D s}$, there are no collisions in the sheath region for lower pressure and low bias operations.

To understand the electrodynamic properties and a variety of chemical and physical phenomena in processing discharges, many computational plasma models have been presented in the literature [8]-[13]. Because the sheath thicknesses are only a few times $\lambda_{D s}$ and the ion mean free path in presheath region could be up to hundreds of $\lambda_{D s}$ in low pressure, high density plasmas, computation of an exact solution is very time consuming. To find a reasonably accurate sheath solution without costing too much in computation, a common approach is to build separate sheath models [9], [14]-[18] for the sheath region and plasma models for the bulk plasma region. The sheath model usually assumes a collisionless sheath without electrons and ionization, while the bulk plasma is assumed electrically quasi-neutral. The boundary (sheath edge) between them is usually set to be the place where the ion velocity is equal to the ion sound speed (Bohm criterion). When the electric field amplitude and space charge density at the boundary are assumed zero, the solution is known as the Child-Langmuir law [19]. Because these three conditions are not, in fact, satisfied at the same location, the assumptions create problems in matching of the solutions of the sheath and the bulk plasma models at the boundary [8], [10], [12]. For a physically accurate solution, not only must the charge densities, plasma potential, and electric field match at the boundary, but their slopes should also be continuous. Many researchers [1], [5], [18] have found the existence of a transition region between presheath and sheath region. 
To match the results of sheath model and plasma model, Godyak and Sternberg [14] have improved the solutions by assuming the electric field to be $k T_{e} / e \lambda_{D s}$ instead of zero at the boundary, even though the choice of this value is still debatable. Treating this problem as a two-scale problem, Riemann [1], [5] and other early researchers [2]-[4] introduced the intermediate scale matching which has been shown to work well for even more collisional plasmas. In addition, Riemann also showed that the Bohm criterion is the right choice for the sheath boundary only if $\varepsilon=\lambda_{D s} / \lambda \ll 1$, where $\lambda$ is the ion mean free path in presheath region.

For collisional plasmas, the definition of the sheath boundary is ambiguous [5], [21]-[23]. When is $\varepsilon$ is small $(O(0.1))$, according to Riemann [5], the Bohm criterion is still good for the sheath boundary. The Child-Langmuir law is often employed to evaluate the sheath thickness of collisionless sheaths. The Child-Langmuir law makes approximations at the sheath edge, such as assuming a step function in the electron density, zero electric field, quasi-neutrality, and negligible ion energy. However, the last assumption is correct only when the sheath potential is much larger than electron temperature $k T_{e} / e$ and the other assumptions are somewhat artificial. Because Poisson's equation is sensitive to these boundary conditions, we examine the effect of these assumptions for sheath thickness evaluation. The normal value of the electrode potential $\phi_{w}$ (relative to the plasma potential) in plasma processing and Langmuir probe operation is in the range of less than several hundred volts. Under these conditions, we raise the question of how high must the bias be for a stepfunction in the electron density at the sheath edge to be a good approximation. Real plasmas are always bounded and somewhat collisional, i.e., $\varepsilon \neq 0$. For the situation of a collisionless sheath, do the collisions in the presheath region affect the sheath thickness? These problems have not been completely addressed to date. Therefore, a more rigorous sheath thickness evaluation for HDP's is necessary.

Presented here is a time-dependent, self-consistent, plasma fluid model to solve for steady state plasma parameter profiles through the whole region including the sheath and presheath region. Unlike previous models, no assumptions are made about the electric field and space charge density at the sheath edge and electron density in the sheath. A semi-analytical sheath solution is also obtained to compare with the fluid model solution. Profiles predicted by the Child-Langmuir law [20] are also calculated for comparison. The intermediate scale matching method [1]-[5] is employed to estimate the electric field and space charge density at the sheath edge, and, therefore, a modified equation for the potential variation in the sheath is attained. Numerical solution for the potential profile given by the modified equation is used to obtain the sheath thickness.

\section{MOdEL DESCRIPTION}

\section{A. Whole Region, Self-Consistent, Plasma Fluid Model}

The unmagnetized bounded plasma fluid model are based on the following set of time-dependent hydrodynamic equations for ions [14]:

$$
\begin{aligned}
n_{i} M\left(\frac{\partial \boldsymbol{u}_{i}}{\partial t}+\boldsymbol{u}_{i}\left(\nabla \cdot \boldsymbol{u}_{i}\right)\right) & =e n_{i} \boldsymbol{E}-k T_{i} \nabla n_{i}-\boldsymbol{F}_{c} \\
\frac{\partial n_{i}}{\partial t}+\nabla \cdot\left(n_{i} u_{i}\right) & =\nu_{i z} n_{e} \\
\nabla^{2} \phi & =e\left(n_{e}-n_{i}\right) / \varepsilon_{o}
\end{aligned}
$$

Here $n_{i}, n_{e}$ denotes the ion and electron densities, $M$ the ion mass, $u_{i}$ the ion velocity, $E$ the electric field intensity, $\nu_{i z}$ the ionization frequency, $\phi$ the electric potential, $e$ the positive elementary charge, $k$ the Boltzmann constant, and $\varepsilon_{0}$ the permittivity of free space. The frictional force $\boldsymbol{F}_{c}$ is caused by collisions and ionization and can be explicitly expressed as $\boldsymbol{F}_{c}=\left(\left|\boldsymbol{u}_{i}\right| / \lambda\right)+\nu_{i z}\left(n_{e} / n_{i}\right) n_{i} M \mid \boldsymbol{u}_{i}$. The first term on the right-hand side is the contribution of ion-neutral collisions and the second term is from ionization. In (1), a scalar pressure is used to approximate the stress tensor for the weakly ionized plasma in which the heat transfer can be neglected, i.e., $\nabla T_{i}=0$ [14]. For simplicity, only the case of direct ionization is considered so the ionization frequency $\nu_{i z}$ is set to be constant. For the same reason, the ion collision mean free path $\lambda$ and ion temperature $T_{i}$ are also assumed constant. $\lambda$ is dependent on the pressure considered while $k T_{i}$ is set as $0.05 \mathrm{eV}$ through this study.

Equation (1) is the momentum equation, (2) is the continuity equation, and (3) is Poisson's equation. In addition, electrons are assumed to satisfy the Boltzmann relation:

$$
n_{e}=n_{e o} e^{e\left(\phi-v_{p}\right) / k T_{e}}
$$

where $V_{p}$ is plasma potential and $n_{e o}$ is the electron density at the center of the bulk plasma. Same as ions, the electron temperature $k T_{e}$ is set as $3 \mathrm{eV}$ through this study.

We restrict the model to one-dimension in space, drop the vector notation for convenience and normalize the variables, yielding, for equations (1)-(4)

$$
\begin{aligned}
\frac{\partial u_{n i}}{\partial t_{n}}+u_{n i} \frac{\partial u_{n i}}{\partial x_{n}} & =-\frac{\partial \phi_{n}}{\partial x_{n}}-T_{n i} \frac{\partial n_{n i}}{\partial x_{n}}-F_{n c} \\
\frac{\partial n_{n i}}{\partial t_{n}}+\frac{\partial\left(n_{n i} u_{n i}\right)}{\partial x_{n}} & =\nu_{n i z} n_{n e} \\
\frac{\partial^{2} \phi_{n}}{\partial x_{n}^{2}} & =n_{n e o} e^{\phi_{n}-V_{n p}}-n_{n i} .
\end{aligned}
$$

The footnote $n$ on variables indicates that they have been normalized by characteristic constants. The normalized variables are $\phi_{n}=e \phi / k T_{e}, u_{n i}=u_{i} / u_{B}, n_{n i}=n_{i} / n_{o}, n_{\text {neo }}=$ $n_{e o} / n_{o}, \nu_{n i z}=\nu_{i z} / \omega_{p i}, \quad F_{n c}=\left|u_{n i}\right| u_{n i} \lambda_{D} / \lambda+$ $\nu_{n i z} n_{e} u_{n i} / n_{i}, V_{n p}=e V_{p} / k T_{e}, T_{n i}=T_{i} / T_{e}, t_{n}=t \omega_{p i}$, and $x_{n}=x / \lambda_{D}$, where $k T_{e}$ is the electron temperature $(\mathrm{eV}), \omega_{p i}=\left(n_{o} e^{2} / M \varepsilon_{o}\right)^{1 / 2}$ is the ion plasma frequency, $u_{B}=\left(k T_{e} / e M\right)^{1 / 2}$ is the Bohm velocity, and $\lambda_{D}=\left(\varepsilon_{o} k T_{e} / n_{o} e^{2}\right)^{1 / 2}$ is the Debye length. The reference value for the plasma density is no, and is chosen to be $10^{11}$ $\mathrm{cm}^{-3}$, a typical value for HDP's.

To solve this one-fluid model, (5)-(7), what we need are the initial conditions of ion density profile and ion velocity profile for (5) and (6) and the boundary conditions of electrode potentials for (7). The input of initial conditions does not 
require a "smart" guess and will be discussed latter. The electrode potentials are all set to zero through this research.

Distinguished from the usual static fluid model [4], [14], this one-fluid model includes the time-dependent term to achieve steady state solutions numerically. The reason for including the time-dependent term is that we can avoid guessing the boundary conditions for ion density and ion velocity in the time-independent forms of the (5) and (6). Any approximation for the boundary conditions will induce errors in the steady state solutions. According to the simulation results, the charge density difference always exists even though the difference is considerably small. It is referred to as a "whole region" solution because the sheath and plasma regions are not calculated separately and hence the matching problem which occurs at the sheath edge for separate model can be avoided. Most importantly, no sheath boundary conditions (like electric field, space charge density), electron density distribution in the sheath region or quasi-neutrality in plasma region are presumed. Those assumptions are all somewhat artificial even though they may be good approximations under some conditions. Furthermore, no assumption has made for the sheath conditions in comparison with the separate model. For instance, the collision, ionization and ion temperature in the sheath region are all included in calculating the solutions. Therefore, with the least assumptions on the initial/boundary conditions and sheath conditions, more accurate calculation results are anticipated with this model. The disadvantage is that more computational time is needed to attain steady state solutions.

To approximate the operating conditions of HDP's, we examined cases with fixed electron temperature $k T_{e}=3$ $\mathrm{eV}$, neutral pressure 3-30 mtorr. We seek a constant plasma generation rate for all cases that results in a plasma density in the typical range for HDP plasmas, $10^{11}-10^{12} \mathrm{~cm}^{-3}$. Therefore, we choose the plasma generation rate equal to the loss rate at the walls corresponding to a Bohm flux for density $n_{O}=10^{11} \mathrm{~cm}^{-3}$. Thus, generation rate, $\nu_{i z} n_{e}$, is set equal to $\left(2 \times n_{o} u_{B}\right) / D \mathrm{~cm}^{-3} \mathrm{~s}^{-1}$ where $D$ is the distance between electrodes. We do not assume quasi-neutrality in plasma region; both $n_{e}$ and $n_{i}$ are determined self-consistently by the balance of loss and generation, as is the plasma potential, $V_{p}$.

\section{B. Semi-Analytic Sheath Model}

To check the accuracy of the fluid model and to find a simpler approach for the evaluation of the sheath thickness and sheath edge location, we have built a more general model of the sheath alone involving the electric field and space charge density at the sheath edge and the electron density in the sheath region. In addition, since solving the time-dependent whole region model is time consuming and hence not practical for a more detailed system simulation, the separate model could be one option if the matching problem (due to incorrect assumptions for sheath boundary conditions and position) can be overcome. This semi-analytic sheath model can be used along with a separate plasma model to save computer time and improve accuracy.
We assume that the sheath regions of HDP's are collisionless with no ionization, cold ions $\left(T_{i}=0\right)$, and Boltzmann electrons. Because of low pressure operation (long ion mean free path) and high plasma density (thin sheath) operation, it is reasonable to assume a collisionless sheath with no ionization. For typical ion temperatures $\left(k T_{i} \cong 0.05 \mathrm{eV}\right)$ and electron temperatures $\left(k T_{e} \cong 3-4 \mathrm{eV}\right)$, cold ions is also a good approximation. By normalizing the charge density to the ion density at sheath edge and choosing the plasma potential reference to be the potential at the sheath edge $\left(V_{n p s}\right)$, the following equation can be obtained from (5)-(7):

$\frac{d \phi_{n}}{d x_{n s}}=\left(2 n_{n e o s}\left(e^{\phi_{n}}-1\right)+2\left(\left(1-2 \phi_{n}\right)^{1 / 2}-1\right)+E_{n o s}^{2}\right)^{1 / 2}$

where $x_{n s}=x / \lambda_{D s}, n_{n e o s}=n_{n e o} \exp \left(V_{n p s}-V_{n p}\right) / n_{n i s}$ is the electron density at the sheath normalized to $n_{n i s}$, the ion density at the sheath edge, and $E_{n o s}$ is the electric field intensity at the sheath edge. normalized to $k T_{e} / e \lambda_{D s}$.

Equation (8) can be readily solved numerically as long as the boundary values $E_{n o s}$ and $n_{n e o s}$ are known. These boundary values can be obtained from either the whole region plasma fluid model or the intermediate scale matching technique discussed later. The former gives precise values but is not practical while the latter provides reasonable estimates for $E_{n o s}$ and $n_{n e o s}$ to substitute in equation (8) to form a modified equation to evaluate sheath thickness.

The well-known Child-Langmuir law makes the following assumptions:

1) cold ions;

2) collisionless sheath region;

3) negligible ion energy at sheath boundary, i.e., $M u_{B}^{2} / 2$ is small in comparison with energy gained in sheath region, (i.e., assuming $k T_{e} / e \ll \phi_{w}$, the potential on wall);

4) zero electric field at the sheath edge;

5) no electrons in the sheath region; and

6) quasi-neutrality in the presheath region.

With these assumptions, (5)-(7) can be combined and solved to give

$$
x_{n s}=\frac{\sqrt{2}}{3}\left(-2 \phi_{n}\right)^{3 / 4}
$$

where we define $x_{n s}=0$ at the sheath edge and $x_{n s}$ increases toward the wall. The comparison of sheath thicknesses determined by the two equations (8) and (9) will be shown later, for the case in which the sheath boundary conditions of (9) are given by the whole region fluid model.

\section{SOLUTION AND DiscUSSION}

In this numerical plasma fluid model, we consider an unmagnetized argon plasma which is bounded by a pair of parallel electrodes. The distance between electrodes is $D=$ $1200 \lambda_{D}$. The computer code is written in Fortran 90 and runs in Fortran 90 Power Station on Windows NT. The ion velocity and ion density are advanced in time by solving the momentum equation (5) and the continuity equation (6). The fourth order Runge Kutta method is used for this task. Poisson's equation 
(7) is solved for the electric potential by the relaxation method. Subroutine SOLVDE adapted from numerical recipes [24] is modified to allow variable grid spacing so that fine and coarse meshes can be applied to the sheath and bulk plasma regions, respectively.

Both space and time are discretized. To obtain acceptable accuracy while maintaining manageable computation times, 673 grid points divide the one-dimensional (1-D) space, $D=$ $1200 \lambda_{D}$ long, with gradually increasing mesh spacing from the electrodes to the center of plasma. The finest mesh spacing $\lambda_{D} / 16$ is next to the electrodes and the coarsest mesh $37 \lambda_{D}$ is at the center. To guarantee a stable solution without numerically induced oscillation, the normalized (by $1 / \omega_{p i}$ ) time step, $\Delta t_{n}$, is required to satisfy $2 \Delta t_{n} / \Delta x_{n} \leq 1$, where $\Delta x_{n}$ is the normalized (by $\lambda_{D}$ ) mesh size. Thus, there is a compromise between accuracy and computation time. The method does not require an initial "smart" guess in order to reach convergence. Instead, the program starts by assuming uniform ion and electron densities, and uniform plasma potential. As soon as an electric potential on the electrodes is applied, the sheath starts to expand. In the early period, a larger time step, a uniform mesh dividing a shorter initial system length, $D_{o}=400 \lambda_{D}$, and a low pass filter are employed to expedite computation. A low pass filter is applied to the ion density profile once every 20 time steps in order to eliminate unstable numerical oscillation in the sheath region. Due to their inertia, ions dominate the dynamic behavior of the numerical plasma oscillation. Additionally, the oscillation wavelength is much shorter than the spatial scale of ion density gradients. Therefore, a low pass filter applied to the ions only is very effective to suppress the unstable oscillation without interfering with the evolution of the sheath. As the steady state is approached, we adopt a variable mesh and smaller time step, gradually increase the length $D$ to $1200 \lambda_{D}$, and eliminate the low pass filter to obtain more accurate solutions. The increasing of $D$ is to lower the frictional force caused by ionization while the ionization rate remains constant as described below. With the assumption of a constant ionneutral collision mean free path, the collision frequency $\nu_{c}$ is proportional to the ion velocity and, therefore, ions collide with neutrals more frequently near the sheath boundary than the center area. The choice of $D=1200 \lambda_{D}$ is to satisfy not only $D \gg \lambda_{D s}$ (sheath scale) but also to ensure that ion-neutral collisions dominate over ionization in the presheath region except near the center area of plasma. The latter condition is in accordance with the situation in real HDP's, where typically the ionization rate is much lower in the presheath region than in the "source" region. On the other hand, Table I shows the collision mean free path, ionization frequency and the position where collision frequency are equal to ionization frequency for different pressure conditions for the argon plasma. The origin $x=0$ is set at the center of the plasma so $x=600 \lambda_{D}$ is at the position of the right electrode. The collision dominated region is much larger than sheath thickness which is at most a couple tens of $\lambda_{D}$ for the range of wall bias potentials of interest.

The whole-region fluid model solutions are all obtained for both walls grounded in this study. The case of $10 \mathrm{mtorr}$ argon pressure is shown in Fig. 1. The whole region view,
TABLE I

The Regions in Which the Ion-Neutral Collision Dominates Ionization in the Fluid Model for Argon Plasma Under Different Pressure. The Center of the Plasma Is at $x=0$ And the Walls Are at $x= \pm 600 \lambda_{D}$

\begin{tabular}{c|c|c|c}
\hline $\begin{array}{c}\text { Pressure } \\
\text { (mTorr) }\end{array}$ & $\lambda(\mathrm{m})$ & $v_{i z}\left(\mathrm{sec}^{-1}\right)$ & Region where $v_{i z} \leq v_{c}$ \\
\hline 3.0 & $1 \times 10^{-2}$ & $5.2 \times 10^{4}$ & $\left|x / \lambda_{D}\right| \geq 137$ \\
\hline 10.0 & $3 \times 10^{-3}$ & $3.9 \times 10^{4}$ & $\left|x / \lambda_{D}\right| \geq 70$ \\
\hline 20.0 & $1.5 \times 10^{-3}$ & $3.1 \times 10^{4}$ & $\left|x / \lambda_{D}\right| \geq 35$ \\
\hline 30.0 & $1 \times 10^{-3}$ & $2.7 \times 10^{4}$ & $\left|x / \lambda_{D}\right| \geq 30$ \\
\hline
\end{tabular}

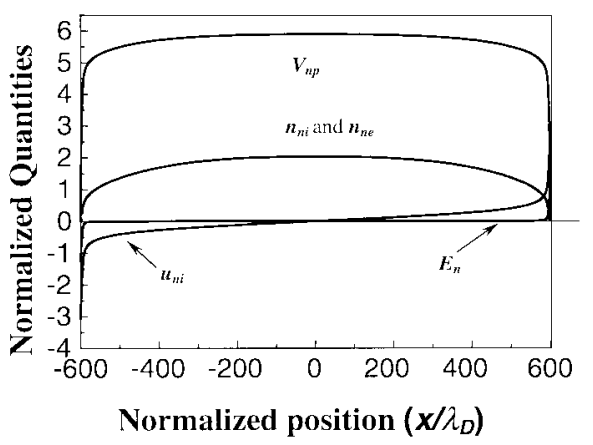

Fig. 1. Whole region solutions of plasma parameters calculated by whole region fluid model for a 10 mtorr argon plasma.

Fig. 1, shows the dramatic variation of plasma parameters in the narrow region close to the wall. The rapid spatial variation is a source of difficulty in computations in obtaining accurate sheath and presheath solutions simultaneously, and the reason that separate models are often adopted.

To show the accuracy of the whole-region solution, the solutions of the sheath model, (8), are compared with those for fluid model for the 3 mtorr case, with the ion temperature, collision, and ionization frequencies set to zero in the sheath edge to be consistent with the assumptions of the sheath model. The definition of the sheath edge is chosen to be the point where the ion velocity reaches the Bohm velocity. During the computation, the sheath edge position is determined at each time step temporarily by the solution of the ion velocity profile at that time. The final correct sheath edge is found as long as the steady state solutions are reached. The sheath boundary conditions (space charge densities and electric field) required as inputs to the sheath model are given by the fluid model. For clarity, the position of the right electrode is set as the origin, the potential at the sheath edge is zero, and the $x$ coordinate and electric field scale are renormalized to the sheath Debye length, $\lambda_{D s}=\left(\varepsilon_{o} k T_{e} / n_{i s} e^{2}\right)^{1 / 2}$ and $k T_{e} / e \lambda_{D s}$, respectively, where $n_{i s}$ is the ion density at sheath edge. In Fig. 2(a)-(d), the close agreement between solutions from the fluid and sheath models indicates the accuracy of the fluid model, even in the sheath region where a much finer mesh is used.

Plotted with the solution of the fluid model (dropping the assumptions that ion temperature, collision, and ionization frequency are zero in the sheath) and sheath model in Fig. 3(a)-(d), the solution of the Child-Langmuir law shows significant differences from the other two. The potential, 


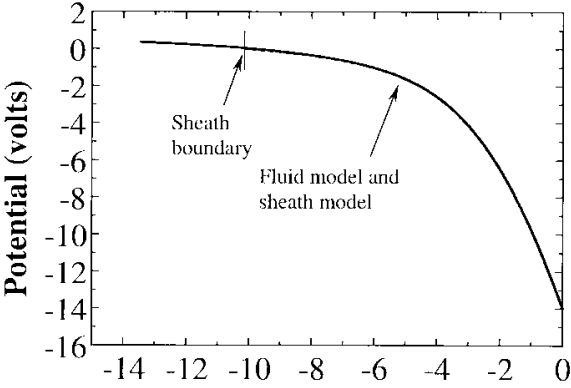

Normalized position $\left(x / \lambda_{D s}\right)$

(a)

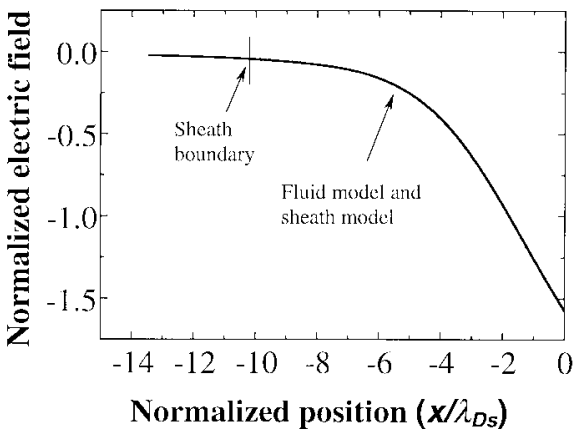

(b)

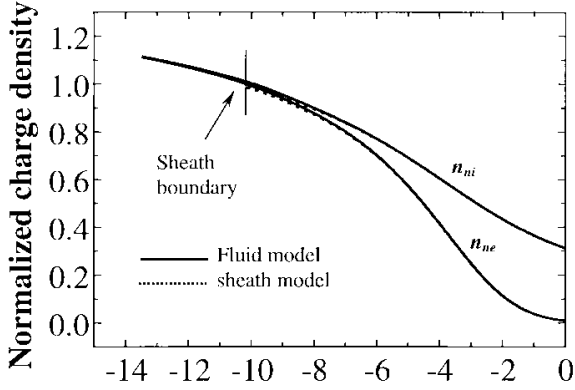

Normalized position $\left(x / \lambda_{D S}\right)$

(c)

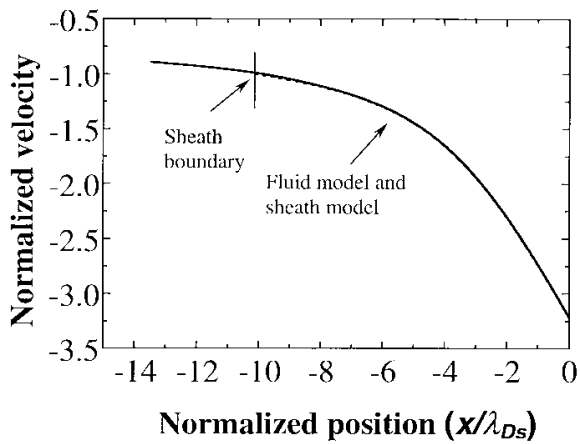

(d)

Fig. 2. Comparison of solutions calculated by the fluid model and sheath model for an argon plasma at a pressure of 3 mtorr. Conditions at the boundary of the sheath region in the fluid model are chosen to be consistent with the assumptions of the sheath model. For the solutions of fluid model, only the sheath region and part of presheath region are plotted: (a) shows the plasma potential profiles, (b) the electric field profiles, (c) the ion and electron density profiles, and (d) the ion velocity profiles in the sheath region. The electric fields are normalized to $k T_{e} / e \lambda_{D s}$, charge densities are normalized to the ion density at the sheath edge $n_{i s}$, ion velocities are normaized to the Bohm velocity $u_{B}$, and position in the sheath is normalized to the Debye length at the sheath edge $\lambda_{D s}$.

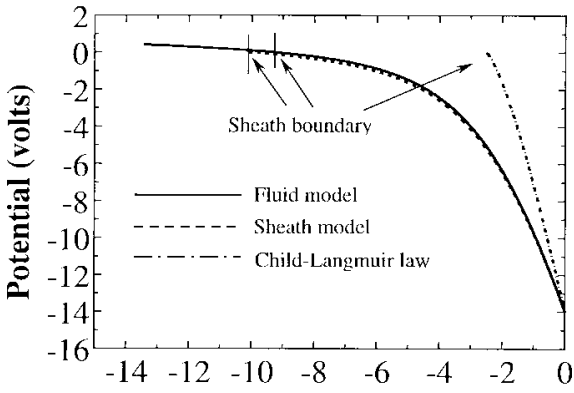

Normalized position $\left(x / \lambda_{D s}\right)$

(a)

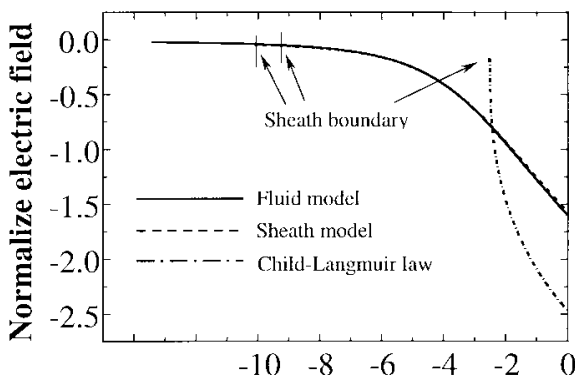

Normalized position $\left(x / \lambda_{D s}\right)$

(b)

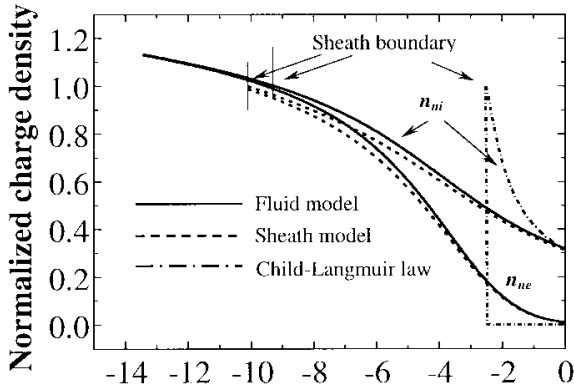

Normalized position $\left(x / \lambda_{D s}\right)$

(c)

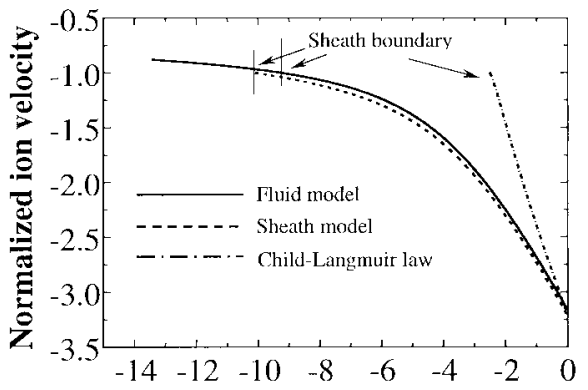

Normalized position $\left(x / \lambda_{D \mathrm{~s}}\right)$

(d)

Fig. 3. Comparison of solutions calculated by the fluid model, sheath model and Child-Langmuir law for argon pressure in pressure 3 mtorr. For the solutions of fluid model, only the sheath region and part of presheath region are plotted. (a) Shows the plasma potential profiles, (b) the electric field profiles, (c) the ion and electron density profiles, and (d) the ion velocity profiles in the sheath region. The electric fields are normalized to $k T_{e} / e \lambda_{D s}$, charge densities are normalized to the ion density at the sheath edgen $n_{i s}$, ion velocities are normalized to the Bohm velocity $u_{B}$, and position in the sheath is normalized to the Debye length at the sheath edge $\lambda_{D s}$. 
electric field, charge density, and ion velocity are shown in Figs. 3(a)-(d), respectively. The discrepancy between the solutions of the fluid model and sheath model is due to different sheath condition assumptions. The fluid model includes the effects of the collision, ionization, and ion temperature while all these are neglected in the sheath model. However, the difference in assumptions does not induce much error in the sheath thickness, in comparison to the sheath thickness evaluated by Child-Langmuir law. This implies the importance of appropriate sheath boundary conditions over simple sheath condition assumptions in correctly estimating the sheath thickness. The sheath thickness (measured from the point where the ion velocity equals the Bohm velocity) given by the fluid model is $10 \lambda_{D s}$ while that of the Child-Langmuir law is $2.5 \lambda_{D s}$ for an electrode potential of $-4.7 T_{e}$ (about -14 volts) relative to the sheath edge. According to Riemann [1], the conditions that electric field and space charge are zero at the sheath edge are valid only when the ratio $\lambda_{D s} / \lambda$ is zero, where $\lambda$ is the ion mean free path in the presheath region. This situation occurs only in semi-infinite collisionless plasmas in which $D$, the size of plasma, is infinite. However, in the real world, laboratory plasmas are always bounded and somewhat collisional.

Instead of using a full region model as presented here, separate models for sheath and bulk plasma region are typically employed to save computer time. However, the matching problem of solutions at the sheath boundary can cause large errors. As shown in Fig. 3(a)-(d), the plasma parameter profiles have substantially different shapes in the whole sheath region for the Child-Langmuir law and fluid model solutions. The errors are inevitable, even with accurate matching of boundary conditions. The discrepancy covers the whole sheath region, making the matching even more difficult. The matching problem is caused by forcing nonphysical values for the electric field and space charge density [Fig. 3(c)] at the sheath boundary position. Note in Fig. 3(c) ion and electron densities are not equal at the sheath edge for the whole region fluid model and for the sheath model. Even though the value of electric field or space charge density may be small, they can make a big difference in the sheath thickness. Thus, the solution of Poisson's equation is very sensitive to the sheath boundary conditions.

The sheath thickness discrepancy is appreciable even for high electrode potential magnitudes as large as 300 and 1000 volts as shown in Fig. 4, determined by the sheath model with the same boundary conditions as Fig. 3(a)-(d). For example, 300 volts bias on the substrate is greater than the self-bias range for almost all cases in plasma etch processing. For this condition, the Child-Langmuir law sheath thickness is $25.1 \lambda_{D s}$ while the fluid model gives the sheath thickness of $36.3 \lambda_{D s}$. The difference is still $31 \%$. For an even higher bias case, 1000 volts, the sheath thickness evaluated using the sheath model is $75.1 \lambda_{D s}$ and using the Child-Langmuir law gives $61.9 \lambda_{D s}$, with an error of $17.6 \%$. Apparently, Fig. 4 depicts that the step-function election density profile assumed in the Child-Langmuir law can be a good approximation only when the bias is sufficiently high. Observe the difference in slope in charge density profiles between these two models

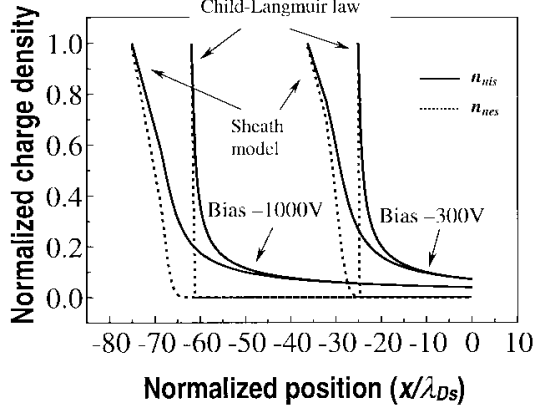

Fig. 4. Comparison of ion and electron density profiles obtained by sheath model and Child-Langmuir law in the sheath region for -300 and -1000 volts biases on the right electrode. The sheath boundary quantities from the fluid model evaluated at the sheath edge (Fig. 3) used as boundary conditions for the sheath model.

TABLE II

Comparison of the Normalized $\left(S_{n}=S / \lambda_{D s}\right)$ Sheath Thickness Calculated by the Fluid Model and Evaluated with Sheath Model and Child-Langmuir Law for Argon Plasma at Different Pressures. The Sheath Edge Is Defined as the Point Where the Ion Velocity Is Equal to the Bohm Velocity

\begin{tabular}{|c|c|c|c|c|}
\hline \multirow{2}{*}{ Pressure } & \multirow{2}{*}{$\lambda \lambda_{J s}$} & \multicolumn{3}{|c|}{ Normalized sheath thickness } \\
\hline & & Fluid model & Sheath model & Child-Langmuir law \\
\hline $\begin{array}{c}3 \text { mTorr } \\
\left(\lambda=1 \times 10^{-2} \mathrm{~m}\right)\end{array}$ & 200 & 9.35 & 10.1 & 2.2 \\
\hline $\begin{array}{c}10 \mathrm{mTorr} \\
\left(\lambda=3 \times 10^{-3} \mathrm{~m}\right)\end{array}$ & 60 & 7.63 & 8.38 & 2.2 \\
\hline $\begin{array}{c}20 \mathrm{mT} \text { orr } \\
\left(\lambda=1.5 \times 10^{-3} \mathrm{~m}\right)\end{array}$ & 30 & 6.58 & 7.31 & 2.2 \\
\hline $\begin{array}{l}30 \text { mTorr } \\
\left(\lambda=10^{-3} \mathrm{~m}\right)\end{array}$ & 20 & 5.95 & 6.67 & 2.2 \\
\hline
\end{tabular}

on the left end of the curves, the transition to the presheath region. The assumptions for sheath boundary conditions in the Child-Langmuir law cannot give the correct slopes at the sheath edge and, therefore, it cannot give the correct sheath thickness values, especially for lower bias conditions.

\section{Sheath THICKNESS AND SHEATH EDGE POSITION EVALUATION}

The assumptions of no collisions, no ionization, and cold ions in the sheath region are based on the conditions of the thin sheath thickness, low electron density in the sheath, and low ion temperature (typical $T_{i}=0.05 \mathrm{eV}$ ). To be more general, all these terms have been included in fluid model to calculate the sheath thickness for different pressures. The results are shown along with the sheath thicknesses found by using the sheath model [(8)] and Child-Langmuir law [(9)] in Table II for comparison. In all cases, the wall potential is close to 14 volts and the sheath Debye length is about $5 \times 10^{-5} \mathrm{~m}$. As expected, the sheath thickness variation shows that it is sensitive to the pressure (or collisions in the presheath region) even though the sheath thickness is still collisionless. Under the assumption of same ion and electron generation rate for each pressure, higher pressure (more collisional) produces a higher plasma density in the discharge center and near the sheath edge. Because the ion loss rates (equal to ion generation rate) at the electrodes must be the same for all pressures, the ion flux must be the same at the sheath edge (due to collisionless sheath with no ionization). The ion densities for all pressures must equal at 
TABLE III

Comparison of Electric Field and Charge Density (Evaluated at the Sheath Edge) Determined by the Fluid Model and Intermediate Scale Matching in Argon Plasmas. The Chosen Cases Correspond to Typical OPERATING CONDITIONS OF HDP's. We CONCLUDE THAT INTERMEDIATE Scale Matching Can Provide Reasonable Estimates for the Electric Field and Space Charge Density at the Sheath Edge

\begin{tabular}{|c|c|c|c|c|c|}
\hline \multirow{2}{*}{$\begin{array}{l}\text { Pressure } \\
\text { (mTorr) }\end{array}$} & \multirow{2}{*}{$\varepsilon=\lambda_{n,} / \lambda$} & \multicolumn{2}{|c|}{$\begin{array}{l}\text { Normalized Electric } \\
\text { Field }\left(E /\left(k T_{,} / e \lambda_{D_{*}}\right)\right)\end{array}$} & \multicolumn{2}{|c|}{$\begin{array}{l}\text { Normalized Space Chargo } \\
\text { Density }\left(\left(n_{i s}-n_{e s}\right) / n_{i s}\right)\end{array}$} \\
\hline & & Fluid Model & Matching & Fluid Model & Matching \\
\hline 3.0 & 0.005 & 0.0488 & 0.04 & 0.0137 & 0.0103 \\
\hline 10.0 & 0.01667 & 0.0887 & 0.0823 & 0.0293 & 0.027 \\
\hline 20.0 & 0.03334 & 0.1333 & 0.1248 & 0.0487 & 0.0471 \\
\hline 30.0 & 0.05 & 0.1702 & 0.1591 & 0.0696 & 0.0652 \\
\hline
\end{tabular}

the sheath edge (defined by the Bohm criterion). As a result, the sheath thickness for the more collisional cases is shorter. This phenomenon is not included in the Child-Langmuir law, which presumes that the sheath thickness depends only on the plasma density at the sheath edge, as depicted in Table II. In addition, the sheath model gives the sheath thickness values much closer to the fluid model than the Child-Langmuir law. Even though there is constant difference about $0.7 \lambda_{D s}$, which results from neglecting collisions and ionization in sheath model, the percentage difference improves as the wall potential become more negative. This is because the electron density in the sheath decreases even further (as shown in Fig. 4) so that the ionization rate in the sheath decreases, and, considering the ion energy dependence of ion-neutral collision cross section, the ion velocity increases, so the ion-neutral collision rate decreases.

We now address the practical problem of how to estimate the sheath thickness and the position of sheath edge without solving the full-blown fluid model. According to the works of Franklin and Ockendon [2] and Riemann [1], [3]-[5], intermediate scale matching provides an appropriate estimate of the electric field and space charge density at the sheath edge for the hydrodynamic model. For the case $\lambda=$ constant, the following electric intensity and charge density guesses are good for the range $2 \times 10^{-4} \leq \varepsilon=\lambda_{D s} / \lambda \leq 1 \times 10^{-2}$ and satisfactory for $\varepsilon=O(0.1)[5]$ :

$$
\begin{aligned}
& E_{\text {nos }} \approx 0.9602\left(\frac{\lambda_{D s}}{\lambda}\right)^{3 / 5} \text { and } \\
& \rho_{\text {space }} \approx 0.7152\left(\frac{\lambda_{D s}}{\lambda}\right)^{4 / 5}
\end{aligned}
$$

where $\lambda$ is the mean free path for ion-neutral collisions in argon. The space charge is defined as $\rho_{\text {space }}=\left(n_{i s}-\right.$ $\left.n_{\text {eos }}\right) / n_{\text {is }}=1-n_{\text {neos }}$. Table III is the comparison of the results of the fluid model with intermediate scale matching in argon plasmas. $\varepsilon$ is in the applicable range of (10) for all chosen conditions covering typical HDP normal operation from 3-30 mtorr. The ion temperature is maintained at $T_{i}=$ $0.05 \mathrm{eV}$. Table III shows that (10) gives reasonable estimates for the electric field and space charge density at the sheath. Fitting the fluid model results using the least square method, gives the following:

$$
\begin{aligned}
& E_{\text {nos }}=1.2204\left(\frac{\lambda_{D s}}{\lambda}\right)^{0.6974}+0.018 \text { and } \\
& \rho_{\text {space }}=0.7262\left(\frac{\lambda_{D s}}{\lambda}\right)^{0.805}+0.0001 .
\end{aligned}
$$

The space charge equation in (11) is almost the same as that in (10) while the exponential factor of electrical field is changed to about 3.5/5 instead of 3/5. Equation (11) is valid only for the pressure range 3-30 mtorr. Substituting (11) into (8), we have a modified equation to estimate the potential variation in the sheath for HDP's

$$
\begin{aligned}
\frac{d \phi_{n}}{d x_{n}} \approx & \left(2\left(1-0.73\left(\frac{\lambda_{D s}}{\lambda}\right)^{4 / 5}\right)\left(e^{\phi_{n}}-1\right)+2\left(1-2 \phi_{n}\right)^{1 / 2}\right. \\
& \left.-2+\left(1.2\left(\frac{\lambda_{D s}}{\lambda}\right)^{7 / 10}+0.018\right)^{2}\right)^{1 / 2}
\end{aligned}
$$

This is a semi-analytic equation and numerical solution is required to obtain the sheath thickness. This equation is distinguished from the Child-Langmuir law by the inclusion of collisions in the presheath region. The other quantities in the sheath region can be determined using (12) with (5) and (6). Equation (11) and modified (12) provide the electric field, space charge density, and sheath boundary position for a model simulation that treats the sheath separately from the rest of the plasma.

\section{SUMMARY}

A time-dependent 1-D plasma fluid model is used to find the whole region solution for a bounded collisionless or weakly collisional plasma and sheath. The sheath thickness under realistic HDP conditions is found to be much thicker than that predicted by the Child-Langmuir law. This is because the assumed sheath boundary conditions (zero electric field and zero space charge density) occur only when the plasma is semi-infinite and collisionless. This also explains the matching problem when plasma and sheaths are treated with separate models. In addition, the sheath thickness depends on collisions in the presheath region even when the sheath itself remains collisionless. The Child-Langmuir law does not account for this influence in the sheath thickness evaluation.

For usual HDP operating conditions in plasma etch processes, intermediate scale matching provides electric field and space charge density estimates at the sheath boundary. Therefore, a modified sheath thickness evaluation equation is obtained which can be used in separate sheath models to save computation time and improve accuracy.

\section{ACKNOWLEDGMENT}

The authors would like to acknowledge Prof. N. Hershkowitz for useful discussions during this work. 


\section{REFERENCES}

[1] K.-U. Riemann, "The Bohm criterion and sheath formation," J. Phys. D: Appl. Phys., vol. 24, pp. 493-518, 1991.

[2] R. N. Franklin and J. R. Ockendon, "Asymptotic matching of plasma and sheath in an active low pressure discharge," J. Plasma Phys., vol. 4, p. $371,1970$.

[3] K.-U. Riemann, "Theory of the sheath edge in a weakly ionized collision dominated plasma," J. Physique Coll., vol. C7, p. 831, 1979.

[4] _ "Kinetic theory of the wall layer in a weakly ionized plasma," J. Nuclear Mater., vol. 76-77, p. 575, 1978.

[5] _ "The influence of collisions on the plasma sheath transition," Phys. Plasmas, vol. 4, no. 11, pp. 4158-4166, 1997.

[6] J. D. Johnson and A. J. T. Holmes, "Edge effect correction for smal planar Langmuir probes," Rev. Sci. Instrum., vol. 61, no. 10, pp 2628-2631, 1990

[7] E. W. McDaniel, J. B. A. Mitchell, and M. E. Rudd, Atomic Collisions: Heavy Particle Projectiles. New York: Willey, 1993.

[8] A. L. Ward, "Effect of space charge in cold-cathode gas discharges," Phys. Rev., vol. 112, no. 6, pp. 1852-1857, 1958.

[9] A. B. Wardlaw and I. M. Cohen, "Continuum analysis of the photoionization chamber in the transition from low to high rates of ionization," Phys. Fluids, vol. 16, no. 5, pp. 637-650, 1973.

[10] J. J. Lowke and D.K. Davies, "Properties of electric discharges sustained by a uniform source of ionization," J. Appl. Phys., vol. 48, no. 12, pp. 4991-5000, 1977

[11] M. J. Kushner, "A kinetic study of the plasma etching process. I. A model for the etching of $\mathrm{S}_{i}$ and $\mathrm{S}_{i} \mathrm{O}_{2}$ in $\mathrm{C}_{n} \mathrm{H}_{m} / \mathrm{H}_{2}$ and $\mathrm{C}_{n} \mathrm{~F}_{m} / \mathrm{O}_{2}$ plasmas," J. Appl. Phys., vol. 53, no. 4, pp. 2923-2938, 1982.

[12] "Monte Carlo simulation of electron properties in RF parallel plate capacitively coupled discharges," J. Appl. Phys., vol. 54, no. 9, pp. 4958-4965, 1983.

[13] D. B. Graves and K. F. Jensen, "A continuum model of DC and RF discharges," IEEE Trans. Plasma Sci., vol. PS-14, pp. 78-91, Apr. 1986.

[14] V. A. Godyak and N. Sternberg, "Smooth plasma-sheath transition in hydrodynamic model," IEEE Trans. Plasma Sci., vol. 18, pp. 159-168, Feb. 1990.

[15] M. A. Lieberman and S. E. Savas, "Bias voltage in finite length, cylindrical and coaxial radio-frequency discharge," J. Vac. Sci. Technol., vol. A8, no. 3, pp. 1632-1641, 1990

[16] T. E. Nitschke and D. B. Graves, "Matching an RF sheath model to a bulk plasma model," IEEE Trans. Plasma Sci., vol. 23, pp. 717-727, Aug. 1995 .

[17] S. A. Self, "Asymptotic plasma and sheath representations for low pressure discharges," J. Appl. Phys., vol. 36, no. 2, pp. 456-459, 1965.

[18] N. Sternberg and V. A. Godyak, "Approximation of the bounded plasma problem by the plasma and the sheath models," Physica, vol. D97, pp. 498-508, 1996.
[19] V. A. Godyak and N. Sternberg, "Dynamic model of the electrode sheaths in symmetrically driven RF discharges," Phys. Rev. A, vol. 42, no. 4, pp. 2299-2312, 1990.

[20] M. A. Lieberman and A. J. Lichtenberg, Principles of Plasma Discharges and Materials Processing. New York: Wiley, 1994, ch. 6, pp. 164-165.

[21] H.-B. Valentini, "Bohm cirterion for the collisional sheath," Phys. Plasmas, vol. 3, no. 4, pp. 1459-1461, 1996

[22] K.-U. Riemann and P. Meyer, comment on "Bohm criterion for the collisional sheath," Phys. Plasmas, vol. 3, no. 12, pp. 4751-4753, 1996

[23] H.-B. Valentini, response to comment on "Bohm criterion for the collisional sheath," Phys. Plasmas, vol. 3, no. 12, pp. 4754-4755, 1996.

[24] W. H. Press, S. A. Teukolsky, W. T. Vetterling and B. P. Flannery, $N u$ merical Recipes in FORTRAN, 2nd ed. Cambridge, U.K.: Cambridge Univ. Press, 1992, ch. 17, pp. 753-763.

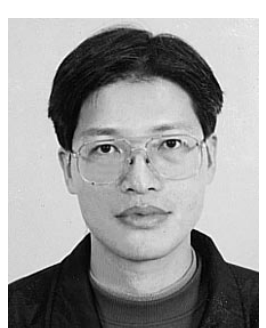

Shiang-Bau Wang was born December 7, 1964, in Hua-Lian, Taiwan, ROC. He received the B.S. and M.S. degrees in nuclear engineering from Tsing Hua University at Tsin-Chu, Taiwan, in 1988 and 1990 , respectively. $\mathrm{He}$ is currently working toward the $\mathrm{Ph} . \mathrm{D}$. degree in electrical and computer engineering at University of Wisconsin-Madison.

After two years of military service as a Lieutenant, he worked with the Second Nuclear Power Plant as a Nuclear Engineer from 1992 to 1995.

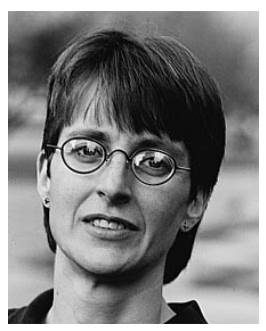

Amy E. Wendt received the B.S. degree in engineering and applied science from the California Institute of Technology, Pasadena, CA, in 1982, and the M.S. and Ph.D. degrees in electrical engineering and computer science from the University of California at Berkeley in 1985 and 1988, respectively.

She joined the Center for Plasma-Aided Manufacturing at the University of Wisconsin-Madison in 1988 as a Research Associate, where she is currently Thrust Area Leader for Plasma Etching. In fall 1990, she joined the Department of Electrica and Computer Engineering at University of Wisconsin-Madison as Assistant Professor, and she was promoted to Associate Professor in 1996. Her research interests include plasma source development, reactor scale-up for processing of large-area substrates, basic mechanisms of plasma physics and chemistry, diagnostic and sensor development for plasma characterization and control, and plasma surface interactions. 